Revista Kinesis, Santa Maria v.36, n.3, p. 53-66, maio-ago. 2018

Centro de Educação Física e Desportos - UFSM

DOI: $105902 / 2316546429722$

Data de submissão: $27-10-2017$

Data de Aceite: 29-11-2018

\title{
COMPARAÇÃO DE VALORES DE FADIGA EM SALTOS VERTICAIS ENTRE MULHERES ADULTAS E ESTUDANTES PRÉ-PÚBERES DE UMA ESCOLA PÚBLICA
}

\author{
COMPARISON OF FATIGUE VALUES IN VERTICAL JUMPS BETWEEN ADULT WOMEN AND PRE-PUBLIC \\ STUDENTS IN A PUBLIC SCHOOL
}

\begin{abstract}
COMPARACIÓN DE VALORES DE FADIGA EN SALTOS VERTICALES ENTRE MUJERES ADULTAS Y ESTUDIANTES PRE-PÚBLES DE UNA ESCUELA PÚBLICA
\end{abstract}

\author{
Samuel Klippel Prusch \\ Samuel_klippel@yahoo.com.br \\ Centro Universitário Cenecista de Osório - UNICNEC \\ Igor Martins Barbosa \\ igormartinsbarbosa2@gmail.com \\ Centro Universitário Cenecista de Osório - UNICNEC \\ Luiz Fernando Cuozzo Lemos \\ luizcanoagem@yahoo.com.br \\ Universidade Federal de Santa Maria - UFSM
}

\begin{abstract}
RESUMO
O objetivo do estudo foi comparar valores de fadiga em saltos verticais entre mulheres adultas e estudantes pré-púberes de uma escola pública. A fadiga muscular foi analisada através da potência de membros inferiores, pelo protocolo de saltos verticais de 30 segundos. Participaram 16 mulheres adultas e 14 estudantes. Para a comparação dos grupos e as variáveis relacionadas aos saltos, foi utilizado o teste $t$ para amostras independentes, com nível $5 \%$ para todos os testes. Os valores de altura de saltos e potência de membros inferiores são afetados pela instauração da fadiga avaliados pelo teste em ambos os grupos.
\end{abstract}

Palavras-Chave: Fadiga; Crianças; Maturação; Adultas

\section{ABSTRACT}

The objective of the study was to compare values of fatigue in vertical jumps between adult women and prepubescent students of a public school. Muscle fatigue was analyzed through the power of lower limbs by the protocol of vertical jumps of 30 seconds. 16 adult women and 14 students participated. For the comparison of groups and variables related to jumps, the t test for independent samples was used, with a level of $5 \%$ for all tests. The values of height of jumps and power of lower limbs are affected by the establishment of fatigue evaluated by the test in both groups.

Keywords: Fatigue; Children; Maturation; Adult

\section{RESUMEN}

El objetivo del estudio fue comparar valores de fatiga en saltos verticales entre mujeres adultas y estudiantes prepúberes de una escuela pública. La fatiga muscular fue analizada a través de la potencia de miembros inferiores, por el protocolo de saltos verticales de 30 segundos. Participaron 16 mujeres adultas y 14 estudiantes. Para la comparación de los grupos y las variables relacionadas con los saltos, se utilizó la prueba t para muestras independientes, con un nivel del $5 \%$ para todas las pruebas. Los valores de altura de saltos y potencia de miembros inferiores se ven afectados por la instauración de la fatiga evaluados por la prueba en ambos grupos. 


\section{Introdução}

A fadiga propriamente dita é um processo subjetivo que abrange aspectos cognitivos, psicológicos e físicos. Podendo ser caracterizado em aguda ou crônica, sendo a primeira classificada quando resulta num grande cansaço físico ou mental, mas que com o repouso a mesma melhora. Já a crônica acontece quando o repouso não gera melhorias, e ainda causa perda da funcionalidade (MCCABE., 2009; MOTA, PIMENTA e FITCH, 2009). A investigação de seus níveis é de grande importância, pois uma maior instauração da fadiga leva a declínios funcionais e, consequentemente, a uma maior dificuldade em sustentar uma atividade com grande carga por pequenos períodos, por exemplo, em práticas esportivas, os sprints, ou em práticas cotidianas, carregar uma caixa de peso elevado, ou pequenas cargas por grandes períodos, como, andar de bicicleta.

Acerca da fadiga física, também denominada como fadiga muscular, esta possui muitos conceitos quanto a sua definição, um destes é, quando não se consegue manter um nível desejado de trabalho ou desempenho numa atividade dinâmica ou isométrica (MANNION e DOLAN, 1996). Outro conceito descrito na literatura sugere que, o começo da ativação muscular voluntária engloba muitos processos que tem como início no controle cortical do cérebro e acaba por terminar nas formações das pontes cruzadas. Assim, é de se pensar que a fadiga ocorra como resultado da falha em qualquer parte dos processos presentes na contração muscular (GANDEVIA, 1998). Portanto, podendo gerar uma diminuição da frequência de ativação muscular, causando uma alteração na propagação do potencial de ação desde a via nervosa, por diversos fatores, dentre eles, motivação, estratégia neural, duração e intensidade da prática, a velocidade de contração, além da continuação de sustentação de uma contração (SANTOS, DEZAN e SARRAF, 2003; ENOKA, 2000).

Historicamente, são distribuídos em duas categorias os fatores que acabam por desenvolver a fadiga. São eles: fatores centrais e periféricos. A fadiga central esta relacionada ao distúrbio na transmissão neuromuscular entre o Sistema Nervoso Central (SNC) e a membrana muscular, podendo ocorrer em vários níveis. Portanto, neste tipo de fadiga uma menor taxa de ativação das unidades motoras acontece, e que geralmente está associada a esforços com maior duração de tempo e de baixa intensidade (SANTOS, DEZAN e SARRAF, 2003; MATOS e CASTRO, 2013; ABD-ELFATTAH, ABDELAZEIM e ELSHENNAWY, 2015).

Já na fadiga periférica as alterações ocorrem dentro do músculo (GIANNESINI, COZZONE e BENDAHAN, 2003; KENT-BRAUM, 1999; SCHILLINGS et al., 2003). Há um decréscimo na força contrátil das fibras dos músculos e existem também alterações nos mecanismos de transmissão de potenciais de ação até o músculo nas junções neuromusculares e nas terminações nervosas (SANTOS, DEZAN e SARRAF, 2003; MATOS e CASTRO, 2013). Ainda sobre a fadiga periférica, vale ressaltar que ela dependerá de 
muitos fatores distintos, como variações dentro do exercício, tipo de fibras recrutadas, nível de treinamento de cada sujeito, dentre outras (ENOKA e STUART, 1992).

Desta forma, uma maneira eficiente de avaliar a fadiga muscular, pode ser vista através de protocolos de testes envolvendo saltos verticais. A partir destes, muitas variáveis podem ser estimadas e estudadas, tais como potência, estimativas de elementos contráteis e índices de fadiga (VALENTE, BATISTA e OLIVEIRA, 2010; BOSCO, LUHTANEN e KOMI, 1983; UGRINOWITSCH et al., 2000). Considerando, estudos sobre a mecânica muscular e os efeitos do pré-alongamento, Carmelo Bosco deu origem a uma bateria de testes, utilizados até a atualidade em todo o mundo, conhecido como "Bosco Tests" (BOSCO et al., 2000; KOMI e BOSCO, 1978). Nessa bateria, se utiliza o salto vertical, o qual se caracteriza por um movimento dinâmico, complexo e com diferentes ativações motoras, que resultam em rápida ação muscular excêntrica, seguida de contração máxima concêntrica (MARCHETTI e UCHIDA, 2011). Esta ação é utilizada como uma das melhores formas para se avaliarem os níveis de potência mecânica muscular, sendo prejudicada diretamente pela fadiga (DAL PUPO, DETANICO e SANTOS, 2012).

Portanto, a fadiga acaba por atuar como um mecanismo de defesa, no qual antes que alguma deterioração em determinada função celular ou orgânica aconteça, esta é ativada, assim evitando possíveis lesões irreversíveis (ROBERGS e ROBERTS, 1997). Desta forma, a especificidade da tarefa, relacionada com as adaptações de forma a retardar a fadiga, deve estar associada às faixas etárias, mais precisamente, em relação a aspectos maturacionais.

Sendo assim, por exemplo, no desenvolvimento infantil as atividades físicas utilizadas na prática, a fim de postergar a fadiga, necessitam ser adequadas de acordo com o nível maturacional das crianças e adolescentes. Anteriormente ao aparecimento dos marcos biológico como, por exemplo, a menarca nas meninas, ambos os sexos nessa fase possuem características similares. No entanto, sabe-se que mulheres maturam geralmente mais cedo, por volta dos 10 aos 12 anos de idade, todavia a consolidação hormonal pode se estender por certo período, valendo ainda ressaltar que o ritmo biológico não representa o cronológico. Contudo, indivíduos que já atingiram a maturação sexual, produzem hormônios, que proporcionarão alterações físicas, comportamentais e psicológicas futuras, havendo assim, demandas e tipologias de exercícios divergentes, conforme o nível maturacional (SILVA, ARAUJO e SOARES, 2012; GALLAHUE e OZMUM, 2005).

Diante disto, o presente estudo tem como objetivo comparar valores de fadiga em saltos verticais entre mulheres adultas e estudantes pré-púberes de uma escola pública.

\section{Materiais e métodos}

O termo de consentimento livre e esclarecido (TCLE) foi assinado pelas mulheres 
adultas. Já as estudantes assinaram um termo de assentimento (TA), no qual explica de forma simples e clara o protocolo utilizado. Além disso, por serem menores de idade, um termo de consentimento livre e esclarecido foi enviado para os responsáveis de cada estudante, para que estes estivessem ciente e livres para autorizar ou não a participação.

O presente estudo foi aprovado pelo Comitê de Ética em Pesquisa do Centro Universitário Cenecista de Osório - UNICNEC sob o protocolo $n^{\circ} 50191115.7 .00005591$.

Os critérios de inclusão foram: não ter sofrido lesão osteomioarticular nos últimos seis meses, assinar o TCLE e/ou TCLE mais TA e, estar praticando atividade física regularmente por pelo menos seis meses, além de uma prática de atividades físicas somadas aos deslocamentos realizados e demais esportes praticados de no mínimo 150 minutos semanais (30 minutos, cinco dias por semana) com intensidade leve a moderada (JAKICIC et al., 2001). Como critério de exclusão foi considerado que as participantes não poderiam apresentar qualquer tipo de distúrbio vestibular, alteração visual sem correção, diabetes, lesões no sistema musculoesquelético ou dor lombar. Além de que, no caso das estudantes, estas não terem tido sua primeira menstruação (Menarca) ainda.

Assim, 30 participantes foram distribuídas em dois grupos, sendo 16 mulheres adultas (GA) e 14 estudantes (GE), selecionados de forma não aleatória, caracterizando uma amostra de conveniência.

As avaliações para o grupo de estudantes ocorreram no ginásio das dependências da Escola Municipal de Ensino Fundamental (Escola Pública) Dezesseis de Dezembro no município de Osório/RS, Brasil. Já para o grupo de mulheres adultas as avaliações ocorreram no Laboratório de Fisiologia do Exercício do Centro Universitário Cenecista de Osório - UNICNEC, Osório/RS, Brasil. Inicialmente, as participantes responderam a uma ficha de avaliação contendo dados demográficos da amostra (idade, massa corporal, altura, presença ou não de lesão, entre outras).

Foram calculados os valores de saltos verticais no início e no fim do protocolo, avaliando altura, potência e potência normalizada, através dos primeiros e dos últimos cinco saltos, de um teste de saltos verticais com duração total de 30 segundos. $O$ protocolo de saltos verticais de 30 segundos (adaptação do teste de Bosco (1983), que tem sua duração de 60 segundos) vem sendo utilizado na literatura (PODERYS et al., 2015; NIKOLAIDIS et al., 2015; DAL PUPO et al., 2014; OKANO et al., 2006). Tornou-se também uma maneira alternativa de se mensurar fadiga muscular, de forma com que o avaliado não tenha um volume grande de tempo saltando, contudo, esse protocolo com tempo reduzido não perde a fidedignidade de seus resultados.

O teste de saltos verticais adaptado teve sua duração reduzida para 30 segundos, porém, não deixou de seguir as orientações de Bosco (BOSCO, LUHTANEN e KOMI, 1983). Neste teste, o sujeito deve realizar um aquecimento livremente durante uns 10 minutos a um trote suave, e depois deve realizar o maior número de saltos verticais possíveis sobre 
uma plataforma de contato CEFISE ${ }^{\circledR}$, que por sua vez estava ligada a um computador que calculou os tempos de voo, as alturas conseguidas e a potência exercida sobre a plataforma (sistema para medida de salto Jump System ${ }^{\circledR}$ ). A técnica aplicada foi a de saltos verticais com contramovimento (Counter Movement Jump), sem ajuda dos membros superiores, de Bosco (BOSCO et al., 2000). Para o protocolo citado anteriormente as voluntárias mantiveram as mãos na cintura e tronco ereto, sem exagerada flexão, joelhos em extensão durante a fase aérea e agachamento de aproximadamente $90^{\circ}$ de flexão durante a fase de contato.

Os dados foram submetidos à estatística descritiva. Foi verificada a normalidade na distribuição dos dados por meio do teste de Shapiro-Wilk, e a homogeneidade por meio do teste de Levene.

Para as comparações na caracterização dos grupos e nas variáveis relacionadas aos saltos, entre os grupos distintos, foi utilizado o teste t para amostras independentes. $O$ pacote estatístico utilizado foi SPSS 22.0. O nível de significância para todos os testes foi de $5 \%$.

\section{Resultados}

A Tabela 1 apresenta dados de caracterização da amostra do presente estudo (Médias e desvios padrão de idade e dados antropométricos, além do número de indivíduos em cada grupo). Na Tabela 2 está ilustrado o nível de significância ( $p$-valor) encontrado com as variáveis de altura dos saltos, potência e potência normalizada, divididas em seus respectivos grupos. Já a Tabela 3 apresenta o nível de significância ( $p$-valor) encontrado com as variáveis percentuais da diferença da altura, potência e potência normalizada, entre os primeiros cinco saltos, com valores dos últimos cinco saltos.

Tabela 1: Médias e desvios padrão de idade e dados antropométricos, além do número de indivíduos em cada grupo.

\begin{tabular}{|c|c|c|c|c|c|}
\hline & \multicolumn{2}{|c|}{ GA $(n=16)$} & \multicolumn{2}{|c|}{ GE $(n=14)$} & \multirow{2}{*}{ p-valor } \\
\hline & $\mathrm{X}$ & $\mathrm{S}$ & $\mathrm{X}$ & $\mathrm{S}$ & \\
\hline Idade (anos) & 21,12 & 3,59 & 9,21 & 1,53 & $<0,001 *$ \\
\hline Estatura (m) & 1,64 & 0,09 & 1,39 & 0,10 & $<0,001 *$ \\
\hline Massa (kg) & 63,98 & 8,46 & 36,61 & 11,05 & $<0,001 *$ \\
\hline
\end{tabular}

GA = Grupo de adultas; GE = Grupo estudantes; *Indica diferença significativa; $\mathrm{p}$ valor: probabilidade de significância; $\mathrm{X}=$ Média; $\mathrm{S}=$ desvio padrão. 
Tabela 2. Variáveis médias da altura dos saltos, potência dos membros inferiores e as probabilidades de significância estatística.

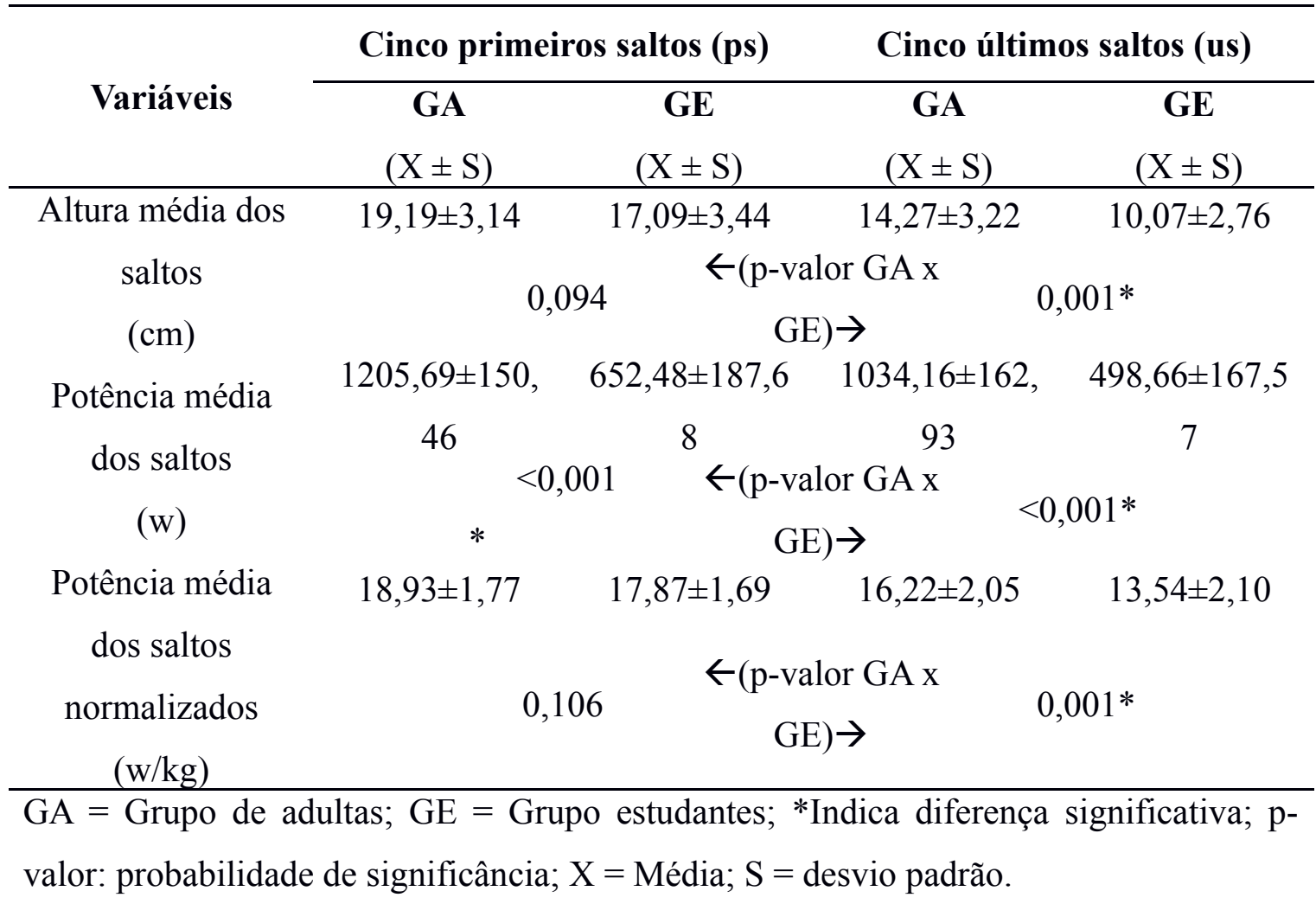

Tabela 3. Variáveis percentuais da diferença (média dos cinco primeiros, e dos cinco últimos saltos) de altura, potência e potência normalizada de membros inferiores e as probabilidades de significância estatística.

\begin{tabular}{cccc}
\hline Variáveis & $\mathrm{GA}$ & $\mathrm{GE}$ & p-valor \\
& $(\mathrm{X} \pm \mathrm{S})$ & $(\mathrm{X} \pm \mathrm{S})$ & \\
\hline Percentual da diferença de altura & $25,22 \pm$ & $41,08 \pm$ & $0,003^{*}$ \\
$(\%)$ & 12,98 & 13,32 & \\
Diferença de potência (\%) & $14,17 \pm 8,73$ & $24,28 \pm 9,20$ & $0,005^{*}$ \\
Diferença de potência normalizada & $14,17 \pm 8,73$ & $24,28 \pm 9,20$ & $0,005^{*}$ \\
$(\%)$ & &
\end{tabular}

GA = Grupo de adultas; GE = Grupo estudantes; *Indica diferença significativa; $\mathrm{p}$ valor: probabilidade de significância; $\mathrm{X}=$ Média; $\mathrm{S}=$ desvio padrão. 


\section{Discussão}

O presente estudo teve como objetivo comparar valores de fadiga em saltos verticais entre mulheres adultas e estudantes pré-púberes de uma escola pública. Na Tabela 1, na comparação entre as adultas e as estudantes foram encontradas diferença estatisticamente significativas em todas variáveis abordadas.

Os principais resultados apresentados pelo presente estudo indicam que entre os grupos, diferenças estatisticamente significativa foram observadas (Tabela 2), para a altura dos últimos cinco saltos, nos valores de potência, tanto nos saltos iniciais como nos finais, além dos valores de potência normalizada nos últimos saltos. Outros resultados encontrados com o estudo quantificaram diferenças estatisticamente significativas, para valores percentuais entre os grupos (Tabela 3), nas variáveis de altura, potência e potência normalizada.

A literatura mostra que só a partir da maturação sexual, um indivíduo terá alterações físicas, comportamentais e psicológicas próximas de um adulto desenvolvido (SILVA, ARAUJO e SOARES, 2012). Indo ao encontro desta afirmação, muitas evidências apontam que logo que se entra no processo de maturação, adolescentes já possuam funcionamento fisiológico similar ao de adultos. Segundo alguns autores, diferenças entre crianças e adultos estariam relacionadas ao perfil metabólico, à ativação das unidades motoras e à composição das fibras musculares (HALIN et al., 2003; DOTAN et al., 2012).

Numa abordagem Fisiológica, a explicação para o aparecimento da fadiga se relaciona com o fato de que para haver produção da força (para saltar) é necessária a concentração de $\mathrm{Ca}^{2+}$ no mioplasma, de uma sensibilidade das proteínas miofibrilares a este íon e de uma produção de força na ativação máxima das pontes cruzadas. Com a menor incidência de $\mathrm{Ca}^{2+}$ para se ligar à troponina, pelo fato de uma queda na concentração de $\mathrm{Ca}^{2+}$ ou sensibilidade reduzida das proteínas contráteis a certa concentração de $\mathrm{Ca}^{2+}$, se gera uma redução na capacidade de produção de força pelos músculos (PEREIRA, 2010).

Diferença estatisticamente significativa foi observada (Tabela 2), para a altura dos últimos cinco saltos, em comparação de grupos. Esses achados podem ser explicados fundamentalmente pela diferença entre crianças e adultos na capacidade anaeróbica (fonte principal utilizada para o protocolo). Em sua obra, Silva et al., (2012) relatam que crianças apresentam uma menor capacidade desta via metabólica. Esta diferença estaria na concentração e na atividade da enzima fosfofrutoquinase, na qual ela é a principal enzima limitadora da glicólise (fenômeno bioquímico relacionado com a atividade anaeróbica). Além disso, as crianças apresentam ainda um menor conteúdo de glicogênio muscular e menor atividade de outra enzima fundamental, a lactatodesidrogenase e, desta forma, nas crianças a glicólise é menos eficaz.

Silva et al., (2012) ainda argumentam que num exercício anaeróbico, ocorre uma acidose metabólica, na qual parte dos prótons de hidrogênio é tamponada no organismo 
através da reação desses prótons de hidrogênio com bicarbonato, resultando num aumento de $\mathrm{CO}_{2}$ na concentração sanguínea, que por sua vez geraria um aumento na ventilação no exercício. Assim, o autor traz em seu estudo, que crianças apresentam uma menor ventilação durante o exercício, portanto, indo ao encontro da afirmação de que exista uma menor produção de energia pela via anaeróbica nas crianças.

Quando se realiza um exercício anaeróbico, ocorre a formação de lactato. A concentração de lactato em crianças aumenta bem menos que a de adultos (DIPLA, TSIRINI e ZAFEIRIDIS, 2009), confirmando a tese de que a glicólise de crianças é menos eficaz. Já a recuperação é mais efetiva depois da maturação sexual. Com essa menor utilização da via anaeróbica, esta seria uma das explicações para inferir-se uma menor produção de altura nos saltos, comparado com adultas.

Ainda na Tabela 2, os valores de potência bruta apresentaram diferenças entre os grupos, tanto nos saltos iniciais, como nos saltos finais. Esses achados de certa forma são evidentes, afinal adultos possuem uma maior maturação física, massa corpórea e estatura estatisticamente significativa (GALLAHUE e OZMUM, 2005). Este melhor desempenho pode ser explicado pelas características dos músculos das crianças, que são diferentes dos adultos. As crianças têm menos massa muscular do que os adultos e, portanto, podem gerar um valor absoluto de energia inferior durante o exercício de alta intensidade (JANSSON, 1996).

Outra possível justificativa pode estar associada com a tipologia de fibras e sua distinta proporcionalidade entre os grupos. Nas quais as fibras musculares diferem em tipo I e tipo II. As fibras do tipo I possuem uma ótima capacidade oxidativa (aeróbia), resposta muscular lenta e resistência à fadiga. Já as fibras do tipo II apresentam capacidade glicolítica (anaeróbia), resposta muscular rápida e pouco resistente (SANTOS, DEZAN e SARRAF, 2003; DOUGLAS, 2002). Metabolicamente, como dito anteriormente, crianças ativariam mais mecanismos oxidativos do que os glicolíticos durante os exercícios e assim gerariam menos substratos (DOTAN et al., 2012; FROST et al., 2012; RATEL, DUCHÉ e WILLIAMS, 2006). Esta diferença poderia ser determinada pela imaturidade do metabolismo glicolítico na infância que proporcionaria uma menor ativação das unidades motoras do tipo II (DOTAN et al., 2012). Embora não haja um consenso na literatura, essa diferente distribuição das fibras, iria ao encontro da afirmação acima (DOTAN et al., 2012; BELL et al., 1980). Dotan et al., (2012) ao avaliar o músculo vasto lateral através de uma dissecação, numa amostra com 22 sujeitos permitiu algumas observações importantes. Segundo os autores, as fibras musculares, de forma geral, são menores nas crianças e a fáscia muscular das crianças não é bem delimitada. Além disso, foi observado que o número total de fibras não mudaria com a idade e, logo, a massa muscular aumentaria pela hipertrofia das fibras.

Com relação à proporção de fibras tipo I e tipo II, os autores sugeriram que haveria uma diferença. As fibras tipo I seriam aos cinco anos de idade maioria (65\%), o que não ocorreu aos 20 anos de idade (50\%), sugerindo uma mudança na tipologia com 
a maturação (transformação de fibras tipo I em tipo II). Assim, é possível inferir que os valores encontrados para o presente estudo, podem ter íntima relação com as mudanças fisiológicas das fibras musculares nas distintas idades, evidenciando os resultados encontrados de potência normalizada, nos quais apenas nos últimos saltos houve diferenças significativas.

Cabe ao profissional de Educação Física, estar sempre atento as suas ações docentes. Com relação ao treinamento de alta intensidade para crianças e adolescentes, Silva et al., (2012) indicam em seus estudos que apesar desse procedimento resultar em uma melhoria do desempenho, também abrevia a vida atlética, bem como pode prejudicar o teto genético das potencialidades de desempenho. Sendo assim, esses dados fisiológicos apoiam a ideia que, pelo menos até metade da adolescência (em torno dos 15 anos de idade), não é recomendado o treinamento de alta intensidade, o qual explora muito a capacidade anaeróbica.

Na Tabela 3 são apresentados os valores percentuais de redução das variáveis analisadas. Na altura dos saltos houve diferença significativa (GA: 25,22\% e GE: 41,08\%), ou seja, GE fadigou mais proporcionalmente que GA.

Pensando nos resultados apresentados acima Silva et al., (2012) evidenciam a importância da capacidade cardiorrespiratória. Na qual os autores apontam para fundamentais diferenças neste sistema, nas quais as crianças possuem um menor débito cardíaco e uma maior frequência cardíaca fazendo com que as crianças atinjam a frequência cardíaca máxima mais rapidamente, em exercícios intensos. Sendo assim, explicando o porquê crianças não têm a capacidade de competir com adultos em exercícios nos quais a fadiga seja determinada principalmente pela oferta de oxigênio aos músculos ativos. Portanto, essa afirmação sugere que num protocolo de fadiga, como o utilizado pelo presente estudo, provavelmente GE atingiram essa frequência máxima cardíaca, num intervalo de tempo anterior do que GA, ou seja, a diferença cardiorrespiratória do início para o final do protocolo para GE seria bem maior. Assim, GE apresentou valores percentuais mais elevados de fadiga que GA, sendo que o fator cardiorrespiratório deve ter participação ativa, juntamente com os fatores já citados anteriormente, para os resultados encontrados.

Por fim, as últimas variáveis a apresentarem diferenças significativamente, foram em valores de potência bruta e normalizada, havendo uma redução de $14 \%$ nas adultas e $24 \%$ nas estudantes. Muitos estudos sugerem que o força muscular no desenvolvimento humano aumentaria gradativamente até os 14 anos para as meninas (MALINA, BOUCHARD e GROWTH, 1991; BOJIKIAN et al., 2005; JOÃO, 2008). Segundo Santos et al., (1991), a maturação funcional da força muscular apresenta valores até 10 anos onde aproximadamente $60 \%$ dos valores finais são encontrados. É sabido que a força aumenta proporcionalmente ao aumento da massa muscular. Porém, além deste fator, a maturação neural desempenha papel fundamental na evolução da força, principalmente em virtude 
da mielinização das fibras nervosas (SILVA, ARAUJO e SOARES, 2012). No entanto, tornase necessário entender que a completa maturação neural só ocorre após a maturação sexual. Ao encontro disto, Silva et al., (2012) confirmam que uma melhoria na coordenação neural está associada a uma melhor sincronização de ativação das unidades motora (com diminuição da ativação dos antagonistas); juntamente com uma melhor coordenação intramuscular (ativação apenas das porções musculares específicas ao movimento).

Ainda associado a essa coordenação neural, as bainhas de mielina possuem suma importância para esse melhor desempenho de força, nas quais, elas por não se desenvolverem plenamente até a puberdade indicam que crianças não são aptas a ganhos de força similares a adultos (SILVA, ARAUJO e SOARES, 2012). Portanto, GE poderia ter uma coordenação não ótima para sustentar sua força. Além disto, é de se pensar que essa não maturação neural, acabaria por gerar um desgaste maior na execução do teste utilizado, no qual uma coordenação intramuscular não tão eficiente proporcionaria um desgaste maior proporcionalmente (início - final) no intervalo de tempo.

\section{Conclusões}

É possível concluir, com base nos resultados apresentados no presente estudo, que valores de altura de saltos e potência de membros inferiores foram afetados pela instauração da fadiga avaliados pelo teste de saltos de 30 segundos, tanto em adultas, como em estudantes pré-púberes.

Em especial, ressalta-se ao fato das estudantes fadigarem em um percentual maior (altura e potência), apontando para uma necessária cautela na atuação do professor de Educação Física, no que diz respeito à utilização exacerbada de atividades de alta intensidade em alunos com sua maturação não completa.

Sugerem-se novos estudos com a utilização da metodologia abordada no presente estudo, a fim de ampliar os conhecimentos acerca da temática.

\section{Referências}

ABD-ELFATTAH, H.M; ABDELAZEIM, F.H; ELSHENNAWY, S. Physical and cognitive consequences of fatigue: A review. Journal of Advanced Research. May; 6(3): 351-358, 2015.

BELL, R.D; MACDOUGALL, J.D; BILLETER, R; HOWALD, H. Muscle fiber types and morphometric analysis of skeletal muscle in six-years-old children. Med Sci Sports Exerc, 12(1): 28-31. 1980.

BOSCO, C; IACOVELLI, M; TSARPELA, O; CARDINALE, M; BONIFAZI, M; TIHANYI, J; VIRU, M; 
DE LORENZO, A; VIRU A. Hormonal responses to whole body vibration in men. European Journal of Applied Physiology, Berlin, Apl; 81(6): 449-454, 2000.

BOSCO, C; LUHTANEN, P; KOMI, P.V. A simple method for measurement of mechanical power in jumping. Eur J Appl Physiol Occup Physiol. 50: 273-82, 1983.

DAL PUPO, J; GHELLER, R; DIAS, J; RODACKI, A; MORO, A; SANTOS, S. Reliability and validity of the 30-s continuous jump test for anaerobic fitness evaluation. Journal of Science and Medicine in Sport. Nov; 17(6): 650-655, 2014.

DAL PUPO, J; DETANICO, D; SANTOS, S.G. Parâmetros cinéticos determinantes do desempenho nos saltos verticais. Rev Bras Cineantropom Desempenho Hum. Aug; 14(1): 41-51, 2012.

DIPLA, K; TSIRINI, T; ZAFEIRIDIS, A. Fatigue resistance during high-intensity irtermittent exercise from childhood to adulthood in males and female. Eur J Appl Physiol. Jul; 106(5): 645-53, 2009.

DOTAN, R; MITCHELL, C; COHEN, R; KLENTROU, P; GABRIEL, D; FALK, B. Child-Adult Differences in Muscle Activation - A Review. Pediatr Exerc Sci, Feb; 24(1): 2-21, 2012.

DOUGLAS, C.R. Tratado de fisiologia aplicada à fonoaudiologia. São Paulo: Robe Editorial; 2002.

ENOKA, R; STUART, D. Neurobiology of muscle fatigue. J Apll Physiol. May; 72(5): 1631-48. 1992.

ENOKA, R.M. Bases neuromecânicas da cinesiologia. 2. ed. São Paulo: Manole, 2000.

FROST, G. DOWLING, J; DYSON, K; BAR-OR, O. Contraction in three age groups of children during treadmill locomotion. J Electr Kinesiol. Sep; 7(3): 179-86. 1997.

GALLAHUE, D.L; OZMUM, J.C. Compreendendo o desenvolvimento motor: bebês, crianças, adolescentes e adultos. 3. ed. São Paulo: Phorte, 2005.

GANDEVIA S.C. Neural control in human muscle fatigue: changes in muscle afferents, motoneurones and motor cortical drive. Acta Physiol Scand, Jul; 163(3): 275-83, 1998.

GIANNESINI , B; COZZONE, P.J; BENDAHAN, D. Non-invasive investigations of muscular 
fatigue: metabolic and electromyographic components. Biochimie. Sep; 85(9): 873-83, 2003.

HALIN, R; GERMAIN, P; BERCIER, S; KAPITANIAK, B; BUTTELLI, O. Neuromuscular response of young boys versus men during sustained maximal contraction. Med Science Sports Exercise, Jun; 35(6): 1042-1048, 2003.

HESPANHOL, J.E; SILVA NETO, L.G; ARRUDA, M; DINI, C.A. Avaliação da resistência de força explosiva em voleibolistas através de testes de saltos verticais. Bras Med Esporte, May/Jun; 13(3):181-4, 2007.

JAKICIC, J.M; CLARK, K; COLEMAN, E; DONNELLY, J.E; FOREYT, J; MELANSON, E. American College of Sports Medicine. ACSM stand position on the appropriate intervention strategies for weight loss and prevention of weight regain for adults. Medicine \& Science in Sports \& Exercise, 33: 2145-2156, 2001.

JANSSON, E. Age-related fiber type changes in human skeletal muscle. In: Maughan RJ, Shireffs SM, editors. Biochemistry of Exercise IX. Champaign (IL): Human Kinetics,: 297307, 1996

JOÃO, F.R. Relação da Aptidão Aeróbia e Muscular com a Composição Corporal, o estado Maturacional e a Atividade Física Habitual de crianças e adolescentes (9-11 anos). Dissertação (Mestrado em Educação Física e Desporto). Universidade de Trás-os-Montes e Alto Douro; Vila Real (Portugal), 2008.

KENT-BRAUM, J.A. Central and peripheral contributions to muscle fatigue in humans during sustained maximal effort. Eur J Appl Physiol, Jun; 80(1):57-63, 1999.

KOMI, P.V. BOSCO, C. Utilization of stored elastic energy in leg extensor muscles by men and women. Medicine and Science in Sports and Exercise, 10(4): 261-5, 1978.

MALINA, R.M; BOUCHARD, C. Growth, Maturation and Physical Activity. Champaign: Human Kinetics Publishers Books, 1991.

MANNION, A.F; DOLAN, P. Relationship between myoelectric and mechanical manifestations of fatigue in the quadriceps femoris muscle group. European Journal of Applied Physiology Occupational Physiology, Berlin, 74(5): 411-419, 1996.

MARCHETTI, P.H; UCHIDA, M.C. Influência da fadiga unilateral de membro inferior sobre o 
salto vertical bilateral. Rev Bras Med Esporte. Nov/Dec; 17(6): 405-8, 2011.

MATOS, C.C; CASTRO, F.A.S. Fadiga: Alterações Fisiológicas e Modelos Conceituais. Rev Brasil Ciências Saúde-USCS. Jul/Set; 11(37): 53-61, 2013.

MCCABE, M. Fatigue in children with long-term conditions: an evolutionary concept analysis. Journal of Advanced Nursing, Oxford. Aug; 65(8): 1735-45, 2009.

MOTA, D.D.C.F; PIMENTA, C.A.M; FITCH, M.I. Pictograma de Fadiga: uma alternativa para avaliação da intensidade e impacto da fadiga. Revista da Escola de Enfermagem da USP. São Paulo, Jan; 43(Esp): 1080-7, 2009.

NIKOLAIDIS, P; ASADI, A; SANTOS, E; GONZÁLEZ, J; PADULO, J; CHTOUROU, H; ZEMKOVA, E. Relationship of body mass status with running and jumping performances in young basketball players. Muscles, Ligaments and Tendons Journal. Jul/Sep; 5(3): 187-194, 2015.

OKANO, A.H; FONTES, E.B; MOREIRA, A; GONÇALVES, E.M; SBRANA, F; TRIANA, R.O; MORAES, A.C. Is There a Dissociation on Electromyographic Signal Response in LowerLimb During 30s Countermovement Jump Test?.Medicine \& Science in Sports \& Exercise Issue. May; 38(5): 447, 2006.

PEREIRA, G.R. Análise da fadiga muscular por meio de duas transformadas tempofrequência. Dissertação (Mestrado em Engenharia Biomédica), Universidade Federal do Rio de Janeiro, Rio de Janeiro (Rio de Janeiro), 2010.

PODERYS, J; GRŪNOVAS, A; PODERIENĖ, K; BULIUOLIS, A; ŠILINSKAS, V; TRINKŪNAS, E. Cardiovascular changes during the performance by nonathletes of Bosco repeated jumps anaerobic test. Medicina. 51(3): 187-192, 2015.

RATEL, S; DUCHÉ, P; WILLIAMS, C.A. Muscle Fatigue during high-intensity exercise in children. Sports Med. 36(12): 1031-65, 2006.

BOJIKIAN, L.P; TEIXEIRA, C.P; BÖHME, M.T.S; RÉ, A.H.N. Relações entre crescimento, desempenho motor, maturação biológica e idade cronológica em jovens do sexo masculino. Rev. bras. Educ. Fís. Esp. Apl/Jun; São Paulo, 19(2): 153-62, 2005.

ROBERGS, R.A; ROBERTS, S.O. Exercise physiology, exercise, performance, and clinical applications. Wbc/ mcgraw-hill. p. 546-563, 1997. 
SANTOS, M.G; DEZAN, V.H; SARRAF, T.A. Bases metabólicas da fadiga muscular aguda. Rev. Bras. Ciên. e Mov, Brasília, Jan; 11(1): 07-12, 2003.

SANTOS, V.C; FIGUEIRA JÚNIOR, A.J; MATSUDO, V.K.R. Porcentagem de maturação e velocidade de crescimento de variáveis antropométricas e neuromotoras de duas regiões distintas. Revista Brasileira de Ciência e Movimento, 5(02): 52-60, 1991.

SCHILLINGS, M.L; HOEFSLOOT, W; STEGEMAN, D.F; ZWARTS, M.J. Relative contributions of central and peripheral factors to fatigue during a maximal sustained effort. Eur J Appl Physiol. Nov; 90(5-6): 562-8, 2003.

SILVA, F.M; ARAÚJO, R.F; SOARES, Y.M. Iniciação esportiva. Medbook, Rio de Janeiro, 2012.

UGRINOWITSCH, C; BARBANTI, V.J; GONÇALVES, A; PERES, B.A. Capacidade dos testes isocinéticos em predizer a "performance" no salto vertical em jogadores de voleibol. Rev. Paul. Educ. Fís. São Paulo. Jul/Dec; 14(2): 172-83. 2000.

VALENTE, A.M; BATISTA, M.T; OLIVEIRA, L.F. Treinamento específico de atletas de voleibol baseado na análise do salto vertical e do índice elástico. Revista mineira de educação física, Viçosa, 18(2): 23-35. 2010. 